\title{
DIAGNÓSTICO DE CRIANÇAS COM ALTERAÇÕES ESPECÍFICAS DE LINGUAGEM POR MEIO DE ESCALA DE DESENVOLVIMENTO
}

\author{
Simone R.V. Hage', Rossana S.S. Joaquim², Karina G. Carvalho², \\ Carlos Roberto Padovani ${ }^{3}$, Marilisa M. Guerreiro ${ }^{4}$
}

\begin{abstract}
RESUMO - Alterações específicas do desenvolvimento da linguagem (AEDL) devem ser identificadas precocemente, pois tais alterações podem interferir nos aspectos sociais e escolares da criança. O objetivo dessa pesquisa foi verificar o desempenho de crianças com diagnóstico de AEDL, em comparação com o de crianças normais, por meio da Escala de Desenvolvimento Comportamental de Gesell e Amatruda (EDCGA). Foram selecionadas 25 crianças de 3 a 6 anos com o diagnóstico de AEDL (grupo estudado - GE) e 50 crianças normais da mesma faixa etária (grupo controle - GC). As crianças do GC apresentaram desempenho satisfatório e melhor que as crianças do GE, em todos os campos da escala. O valor da mediana do GE foi limítrofe nos comportamentos adaptativo e social-pessoal, já no de linguagem foi discrepantemente rebaixado. Concluímos que as alterações de linguagem interferiram na avaliação dos outros campos do desenvolvimento (adaptativo e pessoal-social). Apesar da interferência, a escala pode ser instrumento útil no diagnóstico de AEDL.
\end{abstract}

PALAVRAS-CHAVE: avaliação de linguagem, alterações do desenvolvimento da linguagem, escala de desenvolvimento.

\begin{abstract}
Diagnosis of children with specific language impairment using a developmental scale
ABSTRACT - Specific language impairment (SLI) should have an early diagnosis, since it can interfere with social and school adaptation of the child. The aim of this study was to verify the performance of children with SLI in comparison with normal children using the Behavior Developmental Scale of Gesell and Amatruda. Twenty-five SLI children, 3 to 6 years of age, were evaluated. This group was compared to 50 normal children of the same age. Children of control group showed better performance in all aspects of the scale. The medium value of the studied group was borderline in adaptative and social aspects, and was slightly below the medium in the language aspect. We conclude that language disorder may impair the assessment of other areas of development. Nevertheless, this scale may be useful in the evaluation of children with SLI.

KEY WORDS: language assessment, developmental language disorder, specific language impairment, developmental screening test.
\end{abstract}

Alterações da linguagem podem fazer parte das manifestações de diversos quadros que afetam o desenvolvimento infantil; todavia, há situações em que essas alterações são específicas, não justificadas por alterações mais globais do desenvolvimento ${ }^{1}$. Nestas circunstâncias, o quadro é denominado de alterações específicas do desenvolvimento de linguagem (AEDL)2. AEDL têm amplo espectro, que vão desde um simples atraso que acaba por desaparecer no curso do desenvolvimento, até um distúrbio, com alterações de linguagem persistentes que tendem a afetar a aprendizagem da linguagem escrita ${ }^{1}$. Nos quadros de atraso do desenvolvimento da linguagem (ADL) as manifestações são transitórias, com pouca ou nenhuma repercussão sobre a aprendizagem da linguagem escrita. $O$ atraso costuma respeitar as etapas habituais do desenvolvimento normal da linguagem. Falhas no input são apontadas como causa desse tipo de quadro, como ocorre em situações de otites recorrentes e ambiente pouco favorável para o desenvolvimento da linguagem ${ }^{3}$.Já os quadros de distúrbio do desen-

Estudo realizado na Universidade do Sagrado Coração, Bauru SP, Brasil: ${ }^{1}$ Professora Doutora do Departamento de Fonoaudiologia da Universidade de São Paulo, campus de Bauru; ${ }^{2}$ Fonoaudióloga, especialista em Linguagem pela Universidade do Sagrado Coração; ${ }^{3}$ Professor Titular do Departamento de Bioestatística do Instituto de Biociências da Universidade Estadual Paulista SP Brasil (UNESP); ${ }^{4}$ Professora Associada do Departamento de Neurologia da Universidade Estadual de Campinas (UNICAMP), Campinas SP, Brasil.

Recebido 3 Outubro 2003, recebido na forma final 4 Março 2004. Aceito 6 Abril 2004.

Dra. Simone Hage - Departamento de Fonoaudiologia USP / Campus de Bauru - Alameda Dr. Octávio Pinheiro Brisolla 9-75 - Vila Universitária - 17012-901 Bauru SP - Brasil. E-mail: simonehage@uol.com.br 
volvimento da linguagem (DDL) apresentam alterações mais graves. Intercorrências pré-natais são consideradas fatores de risco para quadros dessa natureza, assim como história familiar para problemas de linguagem ${ }^{4,5}$.

$A E D L$, independente da condição de atraso ou distúrbio, devem ser identificadas precocemente, uma vez que tais alterações podem interferir nos aspectos sociais e escolares do infante ${ }^{6}$. Em geral, os procedimentos de avaliação da linguagem infantil ocorrem por meio de testes padronizados ou de protocolos que envolvem amostra de linguagem oral espontânea e dirigida. Tanto os testes de linguagem como os protocolos são extremamente úteis na verificação de qual ou quais níveis de linguagem estão comprometidos, a saber, fonologia, morfossintaxe, semântica e pragmática. Entretanto, acabam não sendo eficientes quando se quer verificar a existência de discrepância entre o desenvolvimento da linguagem e o de outras áreas do desenvolvimento infantil. Nestas circunstâncias, escalas de desenvolvimento são instrumentos úteis.

As escalas de desenvolvimento refletem os principais ganhos ao longo do desenvolvimento e têm o objetivo de determinar o nível evolutivo específico da criança. O nível de evolução da criança é obtido através de dados relatados sobre seu desenvolvimento e a partir da observação direta de seu comportamento. Algumas escalas avaliam especificamente o desenvolvimento da linguagem, como é o caso da Early Language Milestone Scale $(E L M)^{7}$ e a Reynell Developmental Language Scale $(\text { RDLS })^{8}$. Outras avaliam vários aspectos do desenvolvimento, como a Denver Developmental Screening Test (DDST) ${ }^{9}$ e a Escala de Desenvolvimento Comportamental de Gesell e Amatruda (EDCGA) ${ }^{10}$.

Neste contexto, o objetivo deste estudo foi verificar o desempenho de crianças com AEDL em comparação com crianças normais, por meio da EDCGA, com o intuito de averiguar se alterações de linguagem podem interferir na avaliação de outros campos do desenvolvimento e, ainda, se a utilização dessa escala pode ser instrumento útil no diagnóstico de AEDL.

\section{MÉTODO}

Foram selecionadas 25 crianças de 3 a 6 anos, atendidas nas Clínicas de Fonoaudiologia das Universidades de São Paulo e do Sagrado Coração, ambas em Bauru (SP), cujo prontuário apontava o diagnóstico de AEDL (atraso ou distúrbio). O grupo controle contou com 50 crianças normais também na faixa etária de 3 a 6 anos.

A pesquisa foi aprovada pelo Comitê de Ética em Pesquisa da Universidade do Sagrado Coração (protocolo
35/2002), tendo participado apenas as crianças cujos pais assinaram o termo de consentimento livre e esclarecido.

As crianças foram avaliadas por meio da EDCGA, já que é apontada como uma escala bastante satisfatória, pois as observações realizadas pelos pais podem ser complementadas com as observações do clínico ${ }^{11}$, além de ser acessível no Brasil.

A referida escala avalia o desenvolvimento infantil em 5 campos do comportamento:

- Comportamento adaptativo: observa-se a capacidade da criança quanto à organização dos estímulos, à percepção de relações, à decomposição do todo nas partes e a reintegração dessas partes.

- Comportamento motor grosseiro: avalia-se as reações posturais, o equilíbrio da cabeça, ficar de pé, sentar e andar.

- Comportamento motor delicado: observa-se a capacidade de utilização das mãos e dedos na manipulação de objetos.

- Comportamento de linguagem: observa-se os padrões característicos que fornecem indícios de organização do sistema nervoso central da criança. Engloba-se nesta observação, todas as formas visíveis e audíveis de comunicação, seja através de expressões faciais, gestos, movimentos posturais, vocalizações, palavras, expressões ou frases, incluindo também a capacidade de imitação e compreensão das comunicações de outras pessoas.

- Comportamento pessoal social: observa-se as reações pessoais da criança frente ao ambiente social em que vive, mesmo estando o comportamento pessoal social particularmente sujeito às metas sociais e às variações individuais.

A escala faz uso de tabelas que permite obter um "quociente de desenvolvimento" (QD) em cada um dos 5 campos avaliados e um geral. O QD é a relação entre a idade maturacional (derivada do desempenho comportamental da criança nas provas) e a idade real (cronológica). As tabelas são usadas para checar a presença ou a ausência de comportamentos significativos, representados por sinais de positivo (+) e negativo (-), respectivamente. Tais dados são comparados a uma escala elaborada a partir dos comportamentos padrão apresentados por crianças em determinadas faixas etárias. Foram utilizadas neste trabalho as tabelas das idades chaves de 2, 3, 4 e 5 anos que apresentam colunas adjacentes que abrangem também as idades de 1 ano e seis meses e 6 anos.

A escala descreve que o QD de uma criança perfeitamente media é 100 . Valores entre 85 e 68 seriam considerados limítrofes e teriam implicações em termos de acompanhamento. Valores abaixo de 68 indicariam atraso significativo em uma ou mais áreas do desenvolvimento. Os QDs não medem, mas estimam, o grau de desenvolvimento da criança ${ }^{10}$. Os materiais necessários para o exame do desenvolvimento, para faixa etária abrangida pelas tabelas utilizadas foram confeccionados, desenhados ou comprados, considerando as especificações e os modelos descritos na escala. Dos materiais propostos não foram utilizados apenas os blocos de pesos (por não ter sido possível encontrar material de acordo com as especi- 
ficações), os cartões coloridos e os cartões de comparação estética (por serem específicos do Teste Stanford-Binet). As provas que envolviam estes materiais não foram consideradas na avaliação da criança. A não realização de uma ou outra prova não invalida o exame ${ }^{10}$. O tempo necessário para a aplicação de todas as provas da escala foi 15 a 20 minutos, aproximadamente, dependendo da colaboração da criança, e em apenas uma sessão.

Após a aplicação dos itens das tabelas, foi obtido quociente de desenvolvimento em cada um dos 5 campos avaliados e um QD geral.

A comparação dos grupos (controle e estudado) considerando os 5 campos de desenvolvimento e o somatório destes foi realizada pelo teste não-paramétrico de MannWhitney. Os resultados foram apresentados considerando as medidas de posição (valor mínimo, $1^{\circ}$ e $3^{\circ}$ quartis, mediana, valor máximo) e o valor do teste com o respectivo nível descritivo ("P-value"). A mediana foi a medida escolhida para construir o gráfico por indicar a centralidade da distribuição. Todas as discussões dos resultados foram realizadas no nível $5 \%$ de significância ${ }^{12}$

\section{RESULTADOS}

$\mathrm{Na}$ avaliação do comportamento adaptativo, motor grosseiro, motor delicado e pessoal social, co- mo mostra a Tabela 1, pode-se verificar que o grupo de crianças normais avaliadas apresentou nas medidas descritivas, valor máximo, mediana, primeiro e terceiro quartil, porcentagens iguais a 100. No que diz respeito ao comportamento de linguagem, houve certas dificuldades apresentadas por algumas crianças do grupo controle em estar cumprindo corretamente as provas propostas pela escala, principalmente na tarefa de nomeação de figuras, mas os QDs abaixo de 85\% do comportamento de linguagem dessas crianças não foram considerados significativos, já que os valores da mediana e do primeiro quartil foram iguais a $100 \%$. O QD geral dessas crianças, mesmo para o valor mínimo, foi superior a $85 \%$. As crianças do grupo controle apresentaram desempenho satisfatório na prova de linguagem da escala de desenvolvimento aplicada.

No que se refere às crianças com AEDL, a porcentagem do QD do comportamento adaptativo nos mostrou que a mediana foi $86 \%$, o que significa que as crianças avaliadas atingiram valores próximos ao considerado limítrofe. O mesmo ocorreu com o valor observado no primeiro quartil, que foi

Tabela 1. Medidas descritivas da porcentagem do quociente de desenvolvimento das crianças do grupo controle e estudado e resultados do teste não-paramétrico da comparação dos grupos.

\begin{tabular}{|c|c|c|c|c|c|c|c|}
\hline \multirow[b]{2}{*}{ Campo } & \multirow[b]{2}{*}{ Grupo } & \multicolumn{5}{|c|}{ Medidas Descritivas } & \multirow[b]{2}{*}{$\begin{array}{l}\text { Resultado do } \\
\text { teste estatístico }\end{array}$} \\
\hline & & $\begin{array}{l}\text { *Valor } \\
\text { mínimo }\end{array}$ & $\begin{array}{c}* 1^{\circ} \\
\text { Quartil }\end{array}$ & *Mediana & $\begin{array}{c}* 3^{\circ} \\
\text { Quartil }\end{array}$ & $\begin{array}{l}\text { *Valor } \\
\text { máximo }\end{array}$ & \\
\hline \multirow[t]{2}{*}{ Adaptativo } & Controle & 72 & 100 & 100 & 100 & 100 & $3,80(p<0,0005)$ \\
\hline & AEDL & 33 & 80 & 83 & 100 & 100 & \\
\hline Motor & Controle & 100 & 100 & 100 & 100 & 100 & $4,20(p<0,0001)$ \\
\hline Grosseiro & AEDL & 50 & 75 & 100 & 100 & 100 & \\
\hline Motor & Controle & 100 & 100 & 100 & 100 & 100 & $2,48(p<0,05)$ \\
\hline Delicado & AEDL & 0 & 100 & 100 & 100 & 100 & \\
\hline \multirow[t]{2}{*}{ Linguagem } & Controle & 67 & 100 & 100 & 100 & 100 & $7,79(p<0,0001)$ \\
\hline & AEDL & 0 & 25 & 50 & 50 & 100 & \\
\hline Pessoal & Controle & 67 & 100 & 100 & 100 & 100 & $6,62(p<0,0001)$ \\
\hline Social & AEDL & 25 & 75 & 83 & 88 & 100 & \\
\hline \multirow[t]{2}{*}{ Geral } & Controle| & 87 & 100 & 100 & 100 & 100 & $7,61(p<0,0001)$ \\
\hline & AEDL & 46 & 70 & 79 & 85 & 95 & \\
\hline
\end{tabular}

*os números referem-se às porcentagens dos comportamentos. 


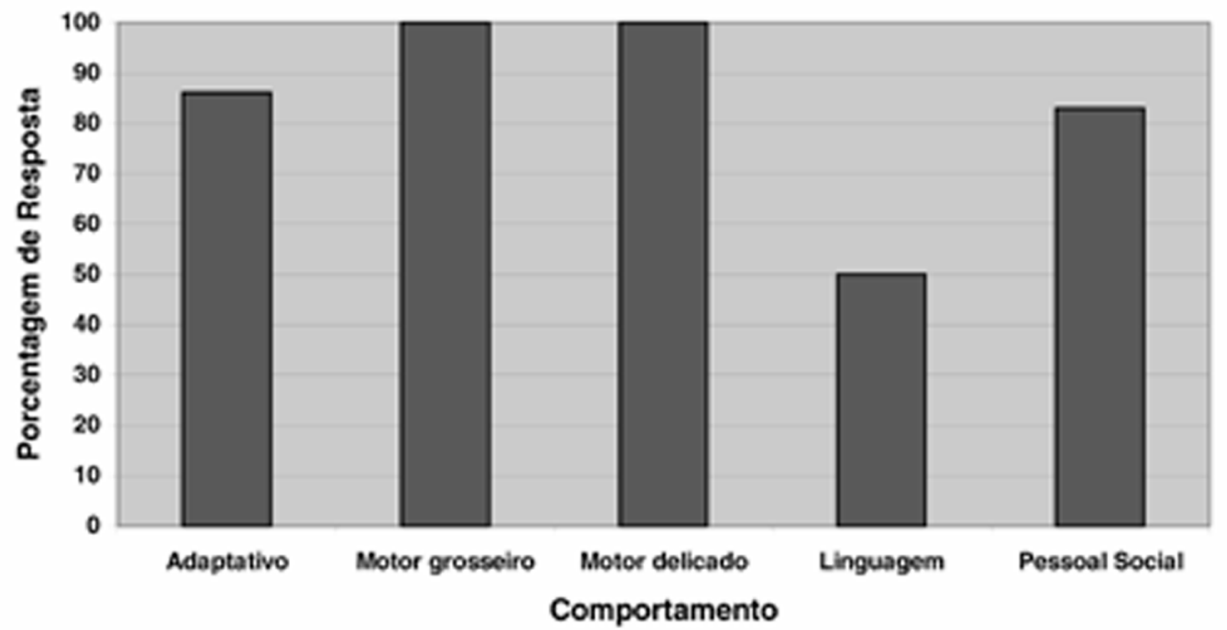

Gráfico 1. Gráfico dos valores da mediana, contendo os Quocientes de desenvolvimento dos 5 campos avaliados das crianças com AEDL.

$80 \%$. As porcentagens estão prejudicadas pelas dificuldades que as crianças avaliadas apresentaram em relação à linguagem. Boa parte das provas em que elas não obtiveram sinal + dependiam da compreensão da linguagem oral para execução.

$\mathrm{Na}$ avaliação do comportamento motor grosseiro e delicado a porcentagem mediana do QD encontrada foi $100 \%$, mostrando que as crianças com AEDL não apresentaram qualquer tipo de dificuldade. As porcentagens mais baixas encontradas no grupo de crianças com AEDL, como mostra a Tabela 1 e o Gráfico 1, estão relacionadas à avaliação do comportamento de linguagem, no qual a porcentagem mediana do QD obtida foi $50 \%$, muito abaixo dos padrões esperados. Tal resultado mostra a discrepância entre os resultados obtidos no comportamento de linguagem em relação aos outros comportamentos.

A porcentagem mediana do QD do comportamento social-pessoal foi $83 \%$, mostrando que crianças apresentaram comportamento pessoal-social limítrofe. O mesmo ocorreu nas porcentagens dos QDs encontrados no primeiro quartil, cujos valores não ultrapassaram $75 \%$. As provas e observações do referido campo avaliam as reações da criança com relação ao ambiente social em que vive. Estas reações estão relacionadas à maturidade neuromotora e à integridade intelectual da criança, mas também solicitam o uso da linguagem na realização das tarefas propostas.

A porcentagem mediana do QD geral foi $79 \%$ e as encontradas no primeiro e terceiro quartil, $70 \%$ e $85 \%$ respectivamente, todas consideradas limítrofes. O QD geral é uma média dos campos avaliados; sendo assim, o desempenho discrepantemente inferior do QD do comportamento de linguagem acabou por rebaixar o QD geral.
O teste não-paramétrico evidenciou diferença significativa em todos os campos avaliados, com desempenho melhor do grupo normal também em todos os campos (Tabela 1).

\section{DISCUSSÃO}

As crianças normais brasileiras avaliadas atingiram porcentagens de QD geral superiores a $85 \%$, apresentando, assim, um desempenho satisfatório na escala de desenvolvimento aplicada. De acordo com a escala utilizada, QD igual a 100 é considerado normal ou perfeitamente médio,. os valores entre 85 e 68 são considerados limítrofes e teriam implicações para acompanhamento e valores abaixo de 68 indicam atraso significativo ${ }^{10}$.

Especificamente quanto ao comportamento linguagem, as crianças normais apresentaram valores do primeiro e terceiro quartil, da mediana e máximo iguais a $100 \%$, evidenciando um bom desempenho nas provas de linguagem. Um dos motivos de se aplicar a escala em crianças normais foi o fato de que diferenças sócio-lingüísticas podem influenciar em testes de linguagem que são padronizados para outras línguas ${ }^{11}$. Todavia, no que se refere as provas de linguagem desta escala, pode-se afirmar que elas são bastante simples e genéricas, envolvendo compreensão de termos espaciais, de ordens situacionais, como "o que você faz quando está com fome?" e nomeação de figuras comuns e rotineiras como cachorro, sapato, casa, relógio, estrela, livro, ação de comer, dormir, chorar. Assim, não parece que as figuras da escala sofram influências sócio-lingüísticas e mereçam adaptação.

O principal critério para o diagnóstico de AEDL 
é a discrepância entre o desenvolvimento de linguagem e o de outras áreas ${ }^{1,2}$. Independente da condição de atraso ou distúrbio, as habilidades de linguagem devem se apresentar no mínimo 12 meses de defasagem ${ }^{3}$. O desenvolvimento motor e cognitivo nestas crianças é normal ou muito próximo do normal ${ }^{3}$. A Tabela 1 e o Gráfico 1 mostram a discrepância entre os resultados obtidos no comportamento linguagem em relação aos outros comportamentos. A porcentagem mediana e do terceiro quartil QDs obtidos foi $50 \%$, muito abaixo dos padrões esperados. A porcentagem encontrada no primeiro quartil foi $25 \%$. Tal resultado está diretamente relacionado às alterações no desenvolvimento da linguagem que as crianças do grupo estudado apresentam.

$\mathrm{Na}$ avaliação do comportamento motor grosseiro e delicado a porcentagem mediana do quociente de desenvolvimento encontrada foi $100 \%$, mostrando que as crianças com AEDL não apresentaram dificuldades nestes campos, apesar da diferença estatisticamente significativa em relação ao grupo controle. Pode-se observar que algumas crianças, três mais precisamente, apresentaram dificuldades em realizar atividades motoras mais refinadas, como segurar pastilhas e colocá-las em uma garrafa, ou contornar o losango duplo com o lápis. Todavia, alguma defasagem psicomotora pode ser encontrada em crianças com AEDL ${ }^{3,5}$.

As porcentagens medianas dos QDs adaptativo e pessoal-social foram $83 \%$, apontando que várias crianças apresentaram comportamento adaptativo e pessoal-social limítrofe. O mesmo ocorreu nas porcentagens dos QDs encontrados no primeiro quartil de ambos os campos, cujos valores não ultrapassaram $80 \%$. Elas apresentaram desempenho estatiscamente significativo pior comparado ao grupo controle.

O que se pode depreender desses resultados, foi que muitas das provas dos campos adaptativo e pessoal-social envolviam habilidades de compreensão ou expressão verbal. Boa parte das provas em que as crianças não obtiveram sinal + dependiam da compreensão da linguagem oral para execução ou de habilidades comunicativo-sociais, como cantar uma música ou cumprimentar. Este fato prejudicou os resultados, como se pode observar na Tabela 1.

Assim, pode-se concluir que alterações no desenvolvimento da linguagem interferiram na avaliação dos outros campos do desenvolvimento pro- postos pela escala, especificamente, os comportamentos envolvidos nos campos adaptativo e pessoal-social. Apesar da interferência, a escala pode ser considerada um instrumento útil no diagnóstico de crianças com possíveis alterações específicas de linguagem, na medida que observou-se uma discrepância entre o desempenho das crianças nas provas do campo da linguagem em relação ao desempenho nos outros campos. O valor da mediana do QD do comportamento de linguagem foi $50 \%$, discrepantemente inferior aos valores da mediana do QD dos outros comportamentos.

O QD geral das crianças com AEDL não pareceu refletir uma estimativa real sobre o desenvolvimento global das mesmas. Por ser uma média dos campos avaliados, o desempenho discrepantemente inferior do QD do comportamento de linguagem, acabou por rebaixar o QD geral, cujo valor da mediana foi inferior a $80 \%$. Assim, uma vez encontrados níveis alterados para o comportamento de linguagem, o nível geral de desenvolvimento não deve ser atribuído.

Um dos desafios dos clínicos que lidam com crianças tem sido o de criar procedimentos que possam diagnosticar o mais precocemente possível alterações do desenvolvimento, com o intuito de estabelecer intervenções mais bem direcionadas. A utilização de escalas de desenvolvimento pode ser um desses procedimentos, permitindo sua utilização por diferentes profissionais envolvidos com o diagnóstico do desenvolvimento.

\section{REFERÊNCIAS}

1. Hall DM, Hill PD. Communication disorders. In Hall DM, Hill PD (Eds). The child with a disability. London: Blackwell, 1996:173-209.

2. Bates E, Thal D, Janowsky JS. Early language development and its neural correlates. In Segalowitz SJ, Rapin I (Eds). Handbook of neurophychology, v. 7. New York: Elsevier, 1992:110-137.

3. Rapin I. Practitioner review: developmental language disorders, a clinical update. J Child Psychol 1996;37:643-655.

4. Tallal P, Ross R, Curtiss S. Familial aggregation in specific language impairment. J Speech Hear Disord 1989;54:167-173.

5. Guerreiro MM, Hage SRV, Guimarães CA, et al. Developmental language disorder associated with polymicrogyria. Neurology 2002;59:245-250.

6. Hage SRV, Guerreiro MM. Distúrbio específico do desenvolvimento da linguagem: subtipos e correlações neuroanatômicas. Pró-Fono - Revista de Atualização Científica 2001;13:233-241.

7. Coplan J, Gleason JR, Ryan R. Validation of an early language milestone scale in a high risk population. Pediatrics 1982;70:677-683.

8. Reynell JK. Escala Reynell para evaluar el desarrollo del lenguaje. Madri: MEPSA, 1985.

9. Frankenburg WK, Dodds JB. The Denver developmental screening test. Pediatrics 1967;71:181-191.

10. Gesell A. Gesell e Amatruda diagnóstico do desenvolvimento: avaliação e tratamento do desenvolvimento neuropsicológico do lactente e da criança pequena, o normal e o patológico. 3.Ed. São Paulo: Atheneu, 2000.

11. Pérez C. Evaluación del lenguaje oral en la etapa 0-6años. Madrid: Siglo Veintiuno, 1995.

12. Norman GR, Streiner DL. Biostatistics: the bare essentials. St. Louis: Nosby-Year Book, 1994 